Letter to the Editor

\section{Mentalization across the psychosis spectrum}

Theory of Mind (ToM) refers to our ability to understand mental states (beliefs and intentions), and to explain behaviors based on these attributions (Premack and Woodruff, 1978). Mentalization is often used as a synonym of ToM, although there are substantial epistemological and historical differences between these concepts. ToM is based on a model emphasizing cognitive attribution of mental states and intentional stances guided by the perception of social signals (e.g., facial expressions) (Dennett, 1987). In contrast, mentalization is a more complex, socially and affectively embedded phenomenon related to reflections on the subjective experience of self and others during interpersonal interactions (Fonagy et al., 2002). The potential relationship between ToM and mentalization, as defined above, is unknown in psychotic disorders. This is a major gap in the literature because measures of ToM and mentalization are seldom differentiated in studies assessing treatment outcomes.

To elucidate this issue, we used the Mentalization Questionnaire (MZQ) for the assessment of subjective experiences, and the Reading the Mind from the Eyes Test (RMET) to investigate ToM. The MZQ is a 15-item self-report questionnaire consisting of four subscales: (1) selfreflection (evasion of thinking about mental states and feelings characterized by a fear of being overwhelmed; e.g., "Most of the time it is better not to feel anything."), (2) emotional awareness (perception and discrimination of inner feelings; e.g., "Often I don't even know what is happening inside of me."), (3) psychic equivalence (blurred boundary between inner mental states and outer reality; e.g. "If I expect to be criticized or offended, my fear increases more and more."), and (4) affect regulation (modulation of emotions, feelings, and impulses; e.g., "Often I can't control my feelings.") (Fekete et al., 2019; Hausberg et al., 2012).

In the RMET, participants saw 36 photographs of eye-regions expressing social emotions and mental states (e.g., playful, embarrassed, and contented). The task was to choose which of four words best described the mental state (Baron-Cohen et al., 2001).

We used a categorical approach based on the DSM-5 diagnoses, as well as a continuum-approach using the schizo-bipolar score (SBS) (Keshavan et al., 2011). Higher SBS values reflect a more schizophrenia-like illness (psychotic and negative symptoms accompanied by functional decline), whereas lower scores indicate bipolar-like psychosis (predominant affective symptoms without a marked functional decline) (Keshavan et al., 2011).

We enrolled 150 patients (schizophrenia, $n=50$; schizoaffective disorder, $n=56$; bipolar disorder, $n=44$ ) who were assessed with the Positive and Negative Syndrome Scale (PANSS), the Hamilton Depression Rating Scale (HAM-D), and the Young Mania Rating Scale (YMRS). All participants gave written informed consent in accordance with the Declaration of Helsinki. The protocol was approved by the Medical Research Council (ETT-TUKEB) (for the details of assessment, see supplementary methods online).
Results from the MZQ are shown in Fig. 1. An analysis of variance (ANOVA) revealed significant main effects of DSM-5 diagnosis ( $F$ $\left.(2,147)=15.35, \eta^{2}=0.17, p<0.001\right)$, MZQ subscales $(\mathrm{F}(3,441)=$ 23.36, $\left.\eta^{2}=0.14, p<0.001\right)$, and a two-way interaction between diagnosis and subscales $\left(\mathrm{F}(6,441)=30.41, \eta^{2}=0.29, \mathrm{p}<0.001\right)$. There were two key findings. First, patients with schizophrenia displayed weaker self-reflection than patients with bipolar disorder (Tukey's Honestly Significantly Difference tests, $p<0.05$ ). Second, patients with bipolar disorder showed the most severe difficulties in the regulation of affect as compared to the other psychotic groups $(p<0.05)$ (Fig. 1.).

As expected, patients with schizophrenia recognized the fewest number of mental states on the RMET $(M=21.0, S D=4.2)$, followed by the schizoaffective group $(M=23.6, S D=3.0)$, and bipolar patients $(M=25.6, S D=4.3)(F(2,147)=16.9, p<0.001)$ (Bora and Pantelis, 2016).

We also performed stepwise regression analyses to explore the predictors of the MZQ scores. The SBS was the most frequent significant predictor (all $p s<0.05$ ). Specifically, higher SBS predicted weaker emotional awareness $(b=0.32)$ and psychic equivalence $(b=0.45)$, whereas the relationship was the opposite in the case of affect regulation $(b=-0.49)$. PANSS negative symptoms predicted dysfunctions in self-reflection $(b=0.26)$ and emotional awareness $(b=0.29)$. Finally, higher HAM-D scores were specifically related to affect regulation $(b=-0.25)$.

Next, we repeated the above-described analyses by adding the RMET scores to the regression models to explore the relationship between mentalization (MZQ) and ToM (RMET). The RMET scores emerged as the single predictor of MZQ emotional awareness $(b=$

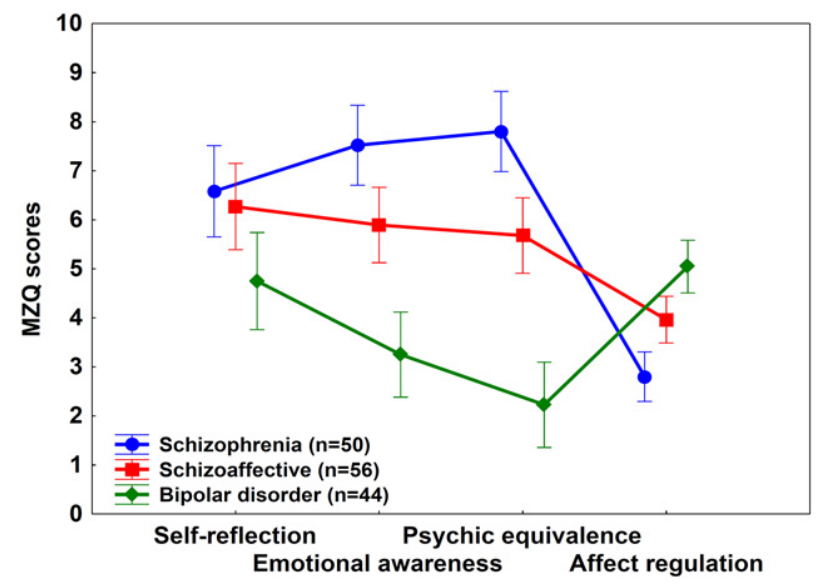

Fig. 1. Mean scores on the Mentalization Questionnaire (MZQ) in patients with psychotic disorders. Error bars show 95\% confidence intervals. Higher scores indicate more difficulties in mentalization. Self-reflection: schizophrenia > bipolar. Emotional awareness and psychic equivalence: schizophrenia $>$ schizoaffective $>$ bipolar. Affect regulation: bipolar $>$ schizoaffective $>$ schizophrenia (Tukey's HSD tests, $p s<0.05$ ). 
$-0.44)$ and self-reflection $(b=-0.41)$. In contrast, the SBS remained the main predictor for psychic equivalence $(b=0.39)$ and affect regulation $(b=-0.56)$ (for the details of the statistical analyses, see supplementary results).

Our results lend support to the assumption that ToM and mentalization are not a unitary phenomenon in psychotic disorders. Specifically, poor self-reflection was related to more severe schizophrenia-like symptoms, whereas weakened affect regulation was associated with dominant bipolar-like symptoms. Patients with schizophrenia-like psychoses demonstrated severe difficulties in discriminating their inner states and exhibited a blurred boundary between their mental representation and the external reality. In this respect, the categorical approach (DSM-5 diagnoses) and the continuum-model (SBS scores) provided consistent results.

Regarding the relationship between ToM and mentalization, ToM strongly predicted emotional awareness and self-reflection, but not psychic equivalence and affect regulation. Therefore, the recognition of mental states of others via social perception is significantly related to thinking about one's own mental states (van der Weiden et al., 2015), but not to reality testing and emotion regulation. These results suggest that distinct dimensions of ToM and mentalization should be taken into consideration in clinical investigations focusing on social cognition and functional remediation in different psychotic disorders (Green et al., 2019).

\section{Role of the funding source}

This work was supported by the BME-Biotechnology FIKP grant of EMMI (BME FIKP-BIO), and by the National Research, Development and Innovation Office (NKFI/OTKA K 128599). The funders had no role in the study design, data collection, analysis, and interpretation, in the preparation of the manuscript, or in the decision to submit the work for publication.

\section{CRediT authorship contribution statement}

Kata Fekete:Conceptualization, Data curation, Formal analysis, Investigation, Methodology, Project administration, Resources, Writing review \& editing.Edina Törölk:Data curation, Formal analysis, Methodology.Zoltán Makkos:Conceptualization, Investigation, Supervision. Oguz Kelemen:Conceptualization, Investigation, Supervision.Katalin Csigó:Conceptualization, Investigation, Supervision, Writing - review \& editing.Szabolcs Kéri:Conceptualization, Formal analysis, Funding acquisition, Methodology, Supervision, Validation, Writing - original draft, Writing - review \& editing.

\section{Declaration of competing interest}

The authors have no conflicts of interest to declare.

\section{Acknowledgements}

We would like to acknowledge and thank our colleagues who assisted with the administrative and technical details of this type of study: Zoltán Kiss, Edit Majoros, and Éva Fehér.

\section{Appendix A. Supplementary data}

Supplementary data to this article can be found online at https://doi. org/10.1016/j.schres.2019.08.023.

\section{References}

Baron-Cohen, S., Wheelwright, S. Hill, J. Raste, Y, Plumb, I., 2001. The "Reading the mind in the eyes" test revised version: a study with normal adults, and adults with Asperger syndrome or high-functioning autism. J. Child Psychol. Psychiatry 42 (2), 241-251.

Bora, E., Pantelis, C., 2016. Social cognition in schizophrenia in comparison to bipolar disorder: a meta-analysis. Schizophr. Res. 175 (1-3), 72-78.

Dennett, D., 1987. The Intentional Stance. MIT Press, Cambridge.

Fekete, K., Torok, E., Kelemen, O., Makkos, Z., Csigo, K., Keri, S., 2019. Dimensions of mentalization in psychotic disorders. Neuropsychopharmacol Hung 21 (1), 5-11.

Fonagy, P., Gergely, G., Jurist, E., Target, M., 2002. Affect Regulation, Mentalization and the Development of the Self. Other Press, New York.

Green, M.F., Horan, W.P., Lee, J., 2019. Nonsocial and social cognition in schizophrenia: current evidence and future directions. World Psychiatry 18 (2), 146-161.

Hausberg, M.C., Schulz, H., Piegler, T., Happach, C.G., Klopper, M., Brutt, A.L., Sammet, I., Andreas, S., 2012. Is a self-rated instrument appropriate to assess mentalization in patients with mental disorders? Development and first validation of the mentalization questionnaire (MZQ). Psychother. Res. 22 (6), 699-709.

Keshavan, M.S., Morris, D.W., Sweeney, J.A., Pearlson, G., Thaker, G., Seidman, L.J., Eack, S.M., Tamminga, C., 2011. A dimensional approach to the psychosis spectrum between bipolar disorder and schizophrenia: the Schizo-bipolar scale. Schizophr. Res. 133 (1-3), 250-254.

Premack, D., Woodruff, G., 1978. Does the chimpanzee have a theory of mind? Behavioral Brain Sciences 1 (4), 515-526.

van der Weiden, A., Prikken, M., van Haren, N.E., 2015. Self-other integration and distinction in schizophrenia: a theoretical analysis and a review of the evidence. Neurosci. Biobehav. Rev. 57, 220-237.

Kata Fekete

Nyírő Gyula National Institute of Psychiatry and Addictions, Budapest, Hungary

Edina Török

Department of Cognitive Science, Budapest University of Technology and Economics, Budapest, Hungary

Zoltán Makkos
Nyírő Gyula National Institute of Psychiatry and Addictions, Budapest, Hungary

Oguz Kelemen

Department of Behavioral Science, University of Szeged, Szeged, Hungary

Katalin Csigó

Nyírő Gyula National Institute of Psychiatry and Addictions, Budapest, Hungary

Szabolcs Kéri

Nyírő Gyula National Institute of Psychiatry and Addictions, Budapest, Hungary Department of Cognitive Science, Budapest University of Technology and Economics, Budapest, Hungary Department of Physiology, University of Szeged, Szeged, Hungary Corresponding author at: Budapest University of Technology and Economics, Department of Cognitive Science, Egry J. str. 1, 1111 Budapest, Hungary.

E-mail addresses: szkeri@cogsci.bme.hu, keri.szabolcs@kzc-opai.hu

24 December 2018 